\title{
Pain imaging in health and disease - how far have we come?
}

\author{
Petra Schweinhardt and M. Catherine Bushnell \\ Department of Anesthesia and Department of Neurology \& Neurosurgery, Faculty of Medicine and Faculty of Dentistry, \\ McGill University, Montreal, Quebec, Canada.
}

\begin{abstract}
Since modern brain imaging of pain began 20 years ago, networks in the brain related to pain processing and those related to different types of pain modulation, including placebo, have been identified. Functional and anatomical connectivity of these circuits has begun to be analyzed. Imaging in patients suggests that chronic pain is associated with altered function and structural abnormalities in pain modulatory circuits. Moreover, biochemical alterations associated with chronic pain are being identified that provide information on cellular correlates as well as potential mechanisms of structural changes. Data from these brain imaging studies reinforce the idea that chronic pain leads to brain changes that could have functional significance.
\end{abstract}

\section{Introduction}

Until the advent of modern noninvasive human brain imaging methodologies about 20 years ago, our understanding of the role of the brain in pain processing was limited. The involvement of the cerebral cortex was disputed, mainly because of the early work of Penfield and Rasmussen, in which pain was seldom evoked by direct cortical stimulation (1). In the last two decades, advances in brain imaging techniques have had a profound influence on our understanding of pain processing. In the early 1990s, human whole-brain functional imaging studies first showed multiple brain areas involved in pain processing (2-4). Other studies have revealed the involvement of forebrain neurotransmitters in pain modulation. New advances in human brain imaging techniques now allow us to better understand functional connectivity in pain pathways, as well as the functional and anatomical alterations that occur in chronic pain patients. Whole brain imaging is now also being applied to small animals, allowing for translation from animal models to man. This review aims to provide an overview of how brain imaging has helped us understand the neural basis of pain processing and pain modulation in healthy individuals and in chronic pain patients.

\section{Defining a pain network in the brain}

There is now a plethora of human brain imaging studies examining cortical and subcortical brain regions involved in acute pain processing in healthy volunteers. Although there are many differences in activation patterns across studies, a consistent cortical and subcortical network has emerged that involves sensory, limbic, associative and motor areas, which some scientists refer to as the "pain matrix" (5). The most commonly activated regions are the primary and secondary somatosensory cortices (S1 and $\mathrm{S} 2$, respectively), anterior cingulate cortex (ACC), insular cortex (IC), prefrontal cortex (PFC), thalamus, basal ganglia, and cerebellum (see Figure 1). As summarized by Bushnell and Apkarian (6), neuroanatomical and electrophysiological studies in rodents and primates confirm that all of these regions receive nociceptive input. In humans, pain-evoked activity in these areas is frequently observed using either activation PET or functional MRI (fMRI)

Conflict of interest: M.C. Bushnell is supported in part by Pfizer Canada. Citation for this article: J Clin Invest. 2010;120(11):3788-3797. doi:10.1172/JCI43498. techniques. Both PET and PMRI measure brain activity indirectly by imaging changes in blood flow, blood oxygenation, or local metabolic changes associated with an increase in neuronal activity. PMRI provides better temporal and spatial resolution than PET and is less costly, so this method is now used more often than PET as an indirect measure of brain activity. Nevertheless, the activation observed in pain-related regions using either technique is consistent with anatomical studies that show probable nociceptive connectivity to these areas $(7,8)$.

The most commonly used fMRI methodology is the blood oxygenation level-dependent (BOLD) technique. However, this method is not ideal for the study of chronic pain states, as it requires a rapidly changing signal that is not always present during chronic, ongoing pain. One way to overcome this limitation is to factor in natural variations in the chronic pain experience (9), but this requires on-line cognitive evaluation and behavioral responses, thus creating possible confounds in data interpretation. Another fMRI technique better suited for evaluating persistent pain involves arterial spin labeling (ASL) (10), which provides a direct measure of blood flow. ASL shows greater sensitivity than BOLD for detecting signals related to stimuli of durations greater than approximately two minutes (11). Owen and colleagues (12) have used ASL to study tonic muscular pain, demonstrating differences in the blood flow time course between brain regions throughout a 15 -minute painful stimulus, with a more rapid return to baseline in the anterior mid-cingulate cortex (aMCC) than in the IC. Since other studies have implicated the aMCC in affective processing of pain (see ref. 7 for review), the authors of that study suggest that this reflects a more rapid decline in the affective response to the painful stimulus. Temporal measurements of pain intensity ratings correlated strongly with the blood flow time course in IC, supporting a role for this region on pain intensity processing. Use of ASL in conjunction with multiple rating scales allows investigators to better understand the temporal relationship between activations in various brain regions and aspects of perception.

\section{Imaging pain-related connectivity}

Researchers are now using imaging techniques to examine connectivity among pain-related regions and possible disruptions of such connectivity in chronic pain patients. One method to evaluate connectivity is to examine the co-variation in activation between a spe- 
A

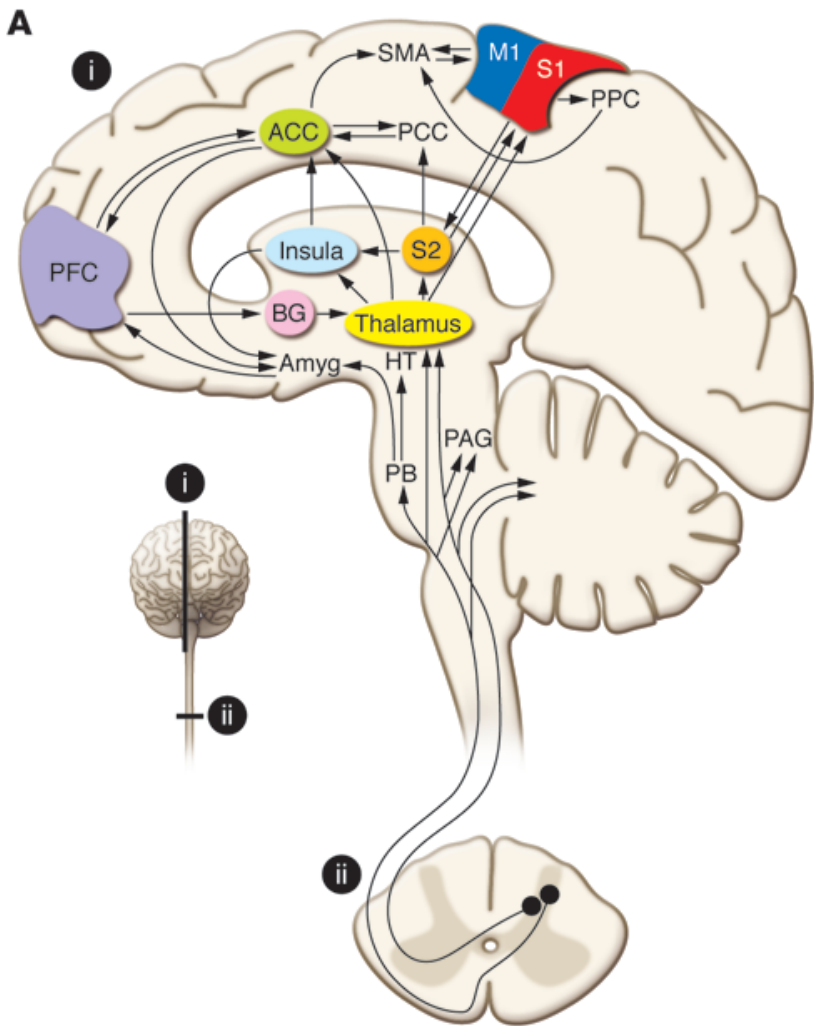

B

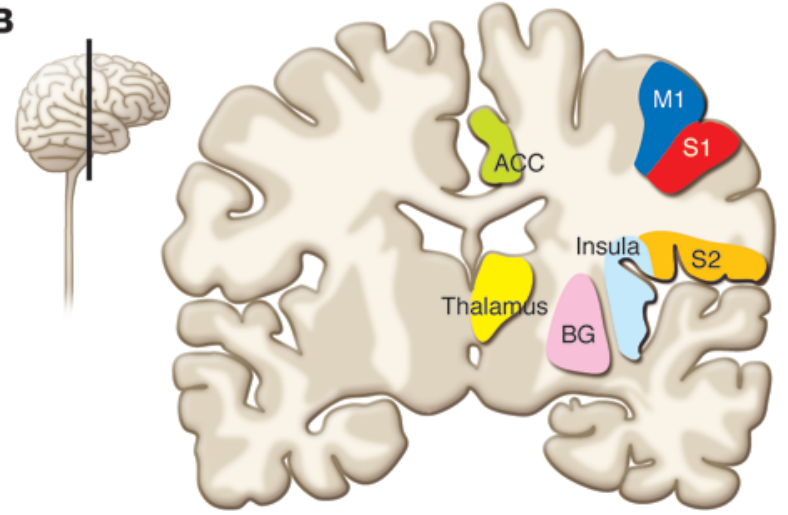

cific area of interest, termed a "seed" area, and other regions in the brain. The simplest way to evaluate such co-variation is to use the activation magnitude of the seed area for each subject as an analytical weighting factor. This method has been used to define regions involved in attentional and emotional modulation of pain (13), as well as in a number of studies of chronic pain patients (see below).

A more refined method to evaluate functional connectivity is to determine those regions for which activation temporally co-varies with the seed region throughout a task performance. In healthy individuals undergoing experimental heat pain, Ploner and colleagues (14) used this method to determine that the individual functional connectivity between the anterior IC and brainstem, regions thought to be related to the subjective perception of pain and the modulation of pain, respectively, predicted whether a potentially noxious event would be perceived as painful.

A direct anatomical measure of brain connectivity uses an MRIbased technique termed "diffusion tensor imaging" (DTI) that

\section{Figure 1}

Ascending pain pathways in the human brain. (A) Schematic representation of ascending pain pathways and brain regions involved in pain processing. (B) The color-coded regions superimposed on an anatomical MRI (coronal slice). Red, S1; orange, S2; green, ACC; light blue, insula; yellow, thalamus; purple, PFC; dark blue, primary motor cortex (M1). SMA, supplemental motor area; PCC, posterior cingulate cortex; BG, basal ganglia; HT, hypothalamus; Amyg, amygdala, PB, parabrachial nuclei. Adapted from European Journal of Pain (7).

can map white matter anatomical connections in the living brain. DTI measures the diffusion of water in the brain and calculates a principal direction of diffusion of water, which is anisotropic in white matter tracts (see ref. 15 for details). Using this technique, Hadjipavlou and colleagues (16) showed white matter tracts connecting regions thought to be involved in descending control of pain, including the frontal cortex and periaqueductal gray matter (PAG) in healthy subjects. DTI is now being used to examine possible disruptions of white matter tracts in chronic pain patients (17), as discussed below.

\section{Spinal cord imaging}

The dorsal horn of the spinal cord has been explored extensively in animal models using in vivo and ex vivo electrophysiological techniques. However, successful imaging of this region in living humans or animals has been elusive because of a number of difficulties. For spinal cord imaging, spatial resolution needs to be at least $1 \mathrm{~mm}$, whereas in the brain, AMRI typically gives a resolution of $2-5 \mathrm{~mm}$. In the spinal cord, there is poor field homogeneity caused by surrounding tissue interfaces, as well as movement artifacts related to pulsations of the cerebral spinal fluid. For the cervical spinal cord, there are the additional problems of respiratory motion and effects of changing lung volume, adding to poor field homogeneity (18). There are now several reports of pain-evoked activity in both the human (19-21) and rat $(22,23)$ spinal cord imaged with fMRI, but the technique still poses some challenges, including signal localization, and so more work needs to be performed before this can become a widespread method of investigation for pain researchers.

\section{Mechanisms underlying psychological modulation of pain}

Psychological factors are known to modulate pain perception in the clinic and in the laboratory (24-26). Nevertheless, the mechanisms underlying such modulation have been difficult to explore in animal studies. The advent of human brain imaging provided an important new avenue for deciphering the neural basis of psychological modulation of pain. In recent years, brain imaging experiments have explored mechanisms underlying attentional and emotional modulation of pain, activity related to expectation and anticipation of pain and, correspondingly, mechanisms of placebo analgesia.

Attention alters nociceptive responsiveness. A number of imaging studies that utilize distracting tasks show modulation of painevoked activity in thalamus and in several cortical regions, including S1, ACC, and IC (13, 27-31). Other regions, including PAG, parts of ACC, and orbitofrontal cortex (within the PFC) have been shown to be activated when subjects perform distracting tasks, thus suggesting that these regions may be involved in the modulatory circuitry related to attention $(30,32-34)$. Nevertheless, most of the distracting tasks require increased cognitive demand, and 


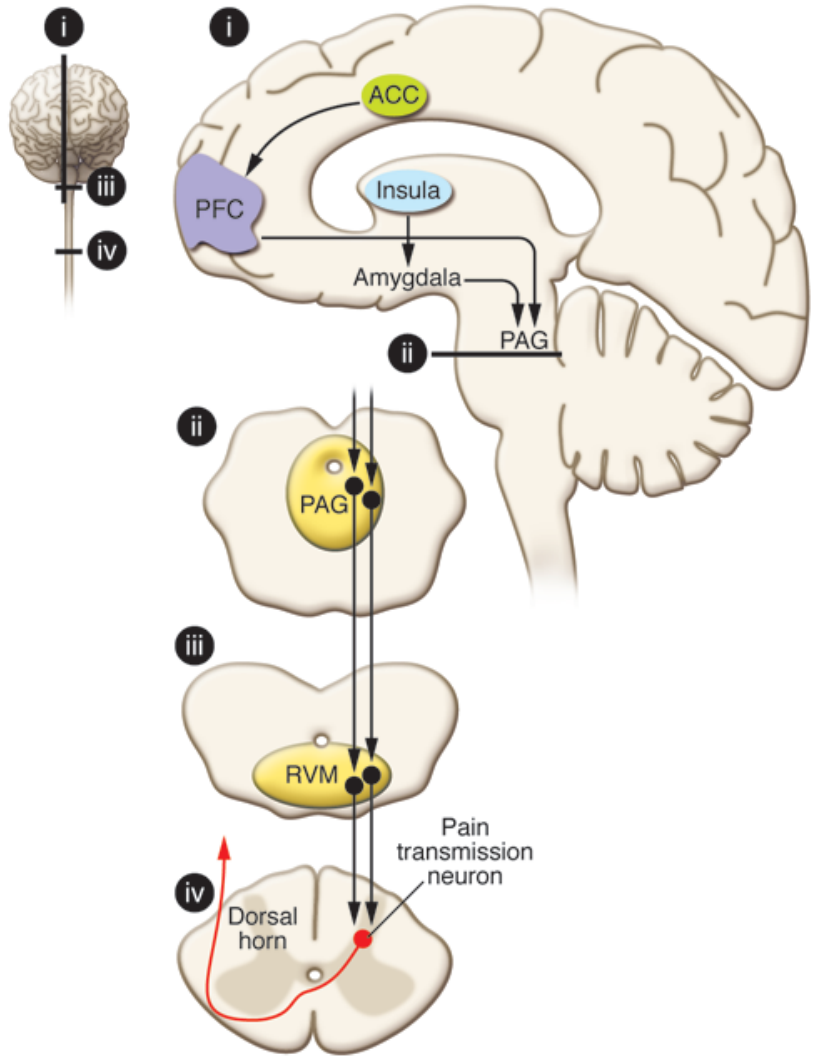

as such, increase arousal and/or alter the emotional state of the subject. Since emotional state can also alter pain $(25,26,35-39)$, it is important to differentiate between modulatory circuits related to attention and those related to emotions.

Emotions alter nociceptive responsiveness. Several studies have used neuroimaging to evaluate the effects of emotional state on pain processing. Phillips and colleagues examined the effect of looking at fearful facial expressions on discomfort, anxiety, and neural activation during non-painful esophageal stimulation in normal subjects (40). Subjects reported increased anxiety and discomfort while viewing the fearful faces, and the negative emotional states enhanced esophageal stimulation-evoked activity in limbic regions such as ACC and IC. Similarly, Villemure and Bushnell (13), using odors to alter mood, showed that a negative mood state led to increased pain-evoked activation in a number of cortical regions, with the largest effect in ACC. Finally, Berna and colleagues (41) found that following the induction of a depressed mood by having subjects read a sad statement and listen to sad music, there was increased pain-evoked activity in frontal areas, ACC, and hippocampus. Together, these studies show that mood state affects pain-evoked activity, particularly in limbic regions of the brain such as the ACC, frontal cortex, and hippocampus (12).

Exploring brain circuits involved in attentional and emotional modulation of pain. Some researchers have suggested that the opiate-sensitive descending pathway from the frontal cortex to the amygdala, PAG, rostral ventral medulla (RVM), and spinal cord dorsal horn (42) (Figure 2) is involved in attentional modulation of pain $(25,28)$. Nevertheless, these studies have usually used tasks that could simultaneously alter attention, arousal and emotional state. Villemure and Bushnell (13) separated attentional state

\section{Figure 2}

Descending pain modulatory pathways that might be involved in psychological modulation of pain. One pathway involves descending input from the ACC to the PFC and then to the PAG. Another descending pathway arrives at the PAG from the insula via the amygdala. $A$ descending pathway from the PAG through the RVM to the dorsal horn of the spinal cord influences nociceptive afferent transmission. Adapted from Science (8) and Nature Reviews Neuroscience (42).

and mood in the same study by having subjects alternate attention between a painful stimulus and a simultaneously presented odor and manipulating mood by having the odor be pleasant or unpleasant. Using functional connectivity analysis, the investigators found that fronto-PAG circuitry is more likely involved in emotional modulation of pain, whereas activation in the superior parietal lobe, which is part of Corbetta and Shulman's proposed "top-down orienting of attention" system (43), is more important for attentional modulation.

Brain responses during anticipation of pain. A particularly interesting observation from fMRI studies is that the anticipation or expectation of pain can activate pain-related areas, such as S1, ACC, and IC, in the absence of a physical pain stimulus (7). Regions thought to be involved in pain modulation, such as PAG, PFC, and ventral striatum, have also been shown to be activated during a period of pain expectation, suggesting that such activation may modulate the impending pain-evoked activation (44-51). In addition, several studies in chronic pain and/or depressed patients show enhanced responses during anticipation of pain, which may be one factor contributing to enhanced pain perception in these patients (52-54).

Anticipation and placebo analgesia. Anticipation of a reduction in pain is a main factor contributing to the placebo effect (24). Neuroimaging studies of expectation-related placebo analgesia show that during the anticipation period immediately preceding the presentation of a noxious stimulus, a cingulo-fronto-PAG descending modulatory pathway is activated, similar to that activated during emotional modulation of pain $(20,55,56)$. Further, using PET to examine the activation of $\mu$-opioid receptors, Wager and colleagues (57) showed that placebo treatment affected endogenous opioid activity in a number of regions, including the PAG, ACC, and lateral PFC. These findings support the idea that activation of the descending modulatory cingulo-fronto-PAG pathway during the expectation of pain relief reduces afferent pain input in the brain. This idea is further supported by an fMRI study examining the influence of naloxone (a $\mu$-opioid antagonist) on placebo-related brain activation (58). The study found that naloxone modulated placebo-induced responses in key structures of the descending pain control system, including the PAG and RVM. Further, naloxone abolished the placebo-induced coupling between rostral ACC and PAG. Functional imaging studies also provide evidence that nociceptive afferent drive at the level of the spinal cord, thalamus, and cortex is reduced during placebo analgesia $(20,55,56)$.

\section{Chronic pain patients have enhanced pain processing}

Despite the demonstration of similarities in pain processing across many types of pain (5), there is evidence that patients with chronic pain syndromes process acute pain differently than do healthy people. Patients with a variety of chronic pain syndromes including back pain, fibromyalgia, irritable bowel syndrome (IBS) and vulvar vestibulitis show higher pain ratings and enhanced pain-evoked neural responses when experimental pain stimuli are 
A

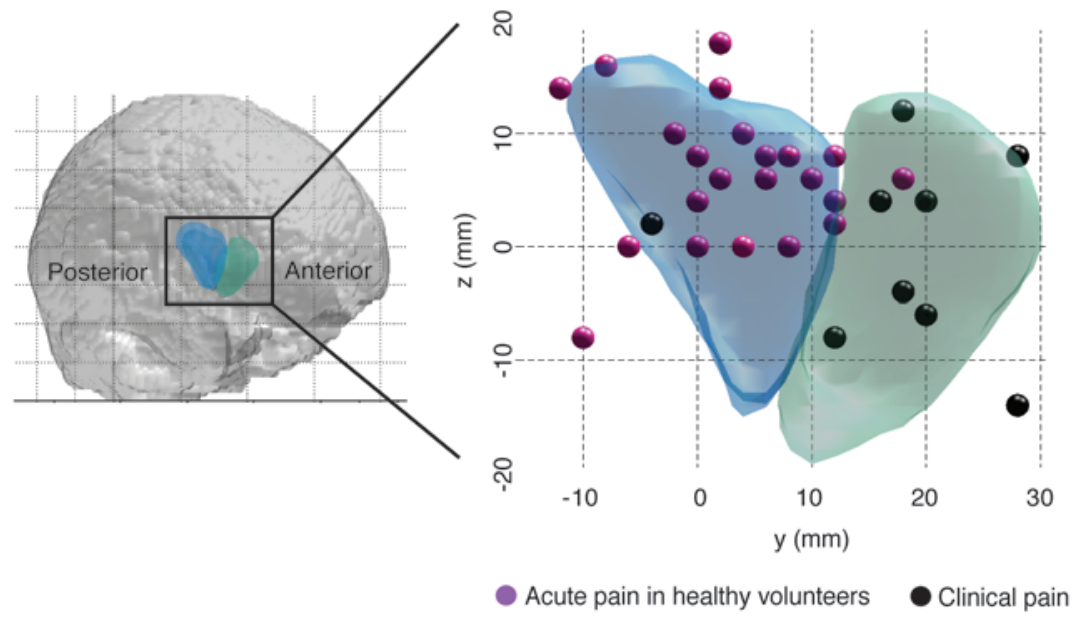

C

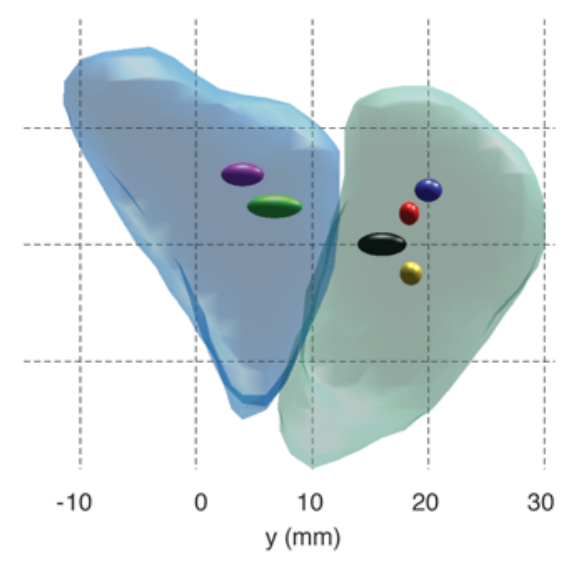

Nonpainful aversive stimuli

Interoception

\section{Figure 3}

Clinical pain is shifted in the insula. (A) Localization of rostral (green) and caudal (blue) anterior insula, as defined in ref. 64, in the human brain. (B) Peak activations found in imaging studies investigating acute experimental pain in healthy subjects (purple spheres) and clinical pain in patients (black spheres). Clinical pain studies investigated ongoing neuropathic pain, provoked mechanical allodynia in neuropathic pain patients, angina pectoris, cluster headache, or punctate hyperalgesia in CRPS. Clinical pain is located significantly more anterior than acute pain (MannWhitney-Wilcoxon test, $P<0.001$ ). (C) The mean localization of anterior insular activation in studies investigating clinical pain (black), acute experimental pain in healthy subjects (purple), interoception (yellow), and anxiety or non-painful stimuli with highly aversive content (red). In addition, cognitive or emotional modulation of acute experimental pain in healthy subjects is depicted (blue). Clinical pain is located as anterior as aversive stimuli or interoception (Mann-Whitney-Wilcoxon tests, $P=0.9$ and $P=0.4$, respectively). Ellipsoids are relative in size to the standard deviation in the $y$ direction. Coordinates are in MNI standard stereotaxic space. $y$ refers to anterior-posterior (nose to back of the head); $z$ refers to superior-inferior (head to feet). Reproduced with permission from Neurolmage (64). Refer to ref. 64 for a complete list of references.

presented to them (59-63). Stimuli that are perceived as non-painful in healthy subjects but as painful in these patients generally activate pain-related areas of the brains in the patients, but not in the healthy individuals $(60,61,63)$. Lawal and colleagues (61) even found enhanced activation to subliminal esophageal stimulation in IBS patients, which suggests that at least part of the enhancement is related to non-psychological factors. These results indicate that the ascending nociceptive signal is amplified somewhere along pain transmission pathways in chronic pain patients, although they do not necessarily imply that the brain augments pain processing. In the following section, we examine the evidence that the brain plays an active modulatory role for pain processing in chronic pain patients.

\section{Do chronic pain patients have altered supraspinal pain modulation?}

Change in spatial representation. Some evidence for altered supraspinal processing comes from the observation that activations related to chronic pain are spatially shifted compared with acute pain or involve brain circuitry not normally activated by acute pain. When closely examining the anterior IC, Schweinhardt and colleagues observed a shift to more rostral regions of the peak representation of neuropathic pain compared with experimental pain (64). This spatial shift to a different functional sub-division might help explain the increased emotional impact chronic pain can have for the individual compared with acute pain: while the caudal anterior IC seems to contain a sensory stimulus representation, the representation of clinical pain in the rostral anterior IC is located in close proximity to affective and interoceptive circuits (Figure 3 ). To investigate whether ongoing pain is processed in different brain areas than acute pain, Baliki and colleagues took an interesting analytical approach (9). They observed that for patients with chronic back pain, although the pain was persistent, there was substantial moment-to-moment variation in the actual intensity of the pain. When brain activations present during periods of rapidly increasing pain were statistically separated from periods of sustained high-level pain, activations present during the rapid pain increase periods were similar to those observed in healthy people during acute pain. Conversely, during periods of high sustained pain, a different brain circuit was engaged involving the PFC and amygdala. Similarly as for the IC activation shift, the recruitment of such areas could underlie the fear and suffering experienced by patients who have endured pain for months or years.

Decreased inbibition versus increased facilitation. Fundamentally, the CNS could augment pain processing in chronic pain patients in two ways: through increased facilitation or through decreased inhibition. In fact, both processes might contribute to enhanced pain perception. It is well known from the animal literature that pathways that can either enhance or inhibit incoming nociceptive signals descend from the brainstem to the spinal cord $(65,66)$. Subsequently, the brainstem has received much attention in human pain imaging studies in recent years. For example, a study in healthy volunteers provides evidence that the brainstem is involved in pain facilitation in a sensitized state (achieved using capsaicin injection) (67). Through use of a parametric design, which allowed equation of pain perception between the sensitized and normal states, the authors could relate the increase in brainstem signal to increased pain perception due to sensitization. Evidence in patients comes from a study of individuals with painful hip osteoarthritis (68) who rated punctate stimuli as "sharper" when applied to the lat- 
eral thigh, i.e., in an area of spinal convergence with the sensory innervation of the hip joint. Increased activation in response to the punctate stimuli was observed in the ACC and dorsolateral PFC, and additionally in the PAG. The magnitude of PAG activation correlated with the degree of patients' neuropathic pain symptoms, as measured by the PainDETECT questionnaire (69). Thus, this study provides evidence that brainstem facilitatory mechanisms might play a role for neuropathic symptoms in a disorder that has been traditionally considered a nociceptive condition.

Although the brainstem and descending pathways are likely to be important pain modulators in humans, it should be emphasized that some pain modulatory circuitry probably operates strictly supra-spinally, as suggested by attentional modulation in healthy volunteers (13). The medial PFC appears to be an important site of supra-spinal pain facilitation. Rheumatoid arthritis patients were shown to activate the medial PFC exclusively in response to clinically relevant pain and not in disease-irrelevant pain (70). Interestingly, the magnitude of depressive symptoms of the largely not clinically depressed patients were positively related to the medial PFC activation. Moreover, the magnitude of medial PFC activation correlated with activation in several other supra-spinal structures, including the hippocampal complex, which has been shown to play an important role in pain augmentation in healthy volunteers by negative affect $(13,71)$. Importantly, medial PFC hyperactivity was related to a measure of the patients' clinical pain that partly accounted for systemic inflammation, indicating that the medial PFC was indeed involved in enhancing clinical pain. In accordance with a pain facilitatory role, activity in the medial PFC in IBS patients has been shown to disrupt a functional connection between the lateral PFC and the PAG (72), two areas that have been implicated in endogenous pain inhibition. As discussed above, the PAG also plays a role in pain facilitation, and it is important to point out that fMRI cannot differentiate between activation in brainstem facilitatory and inhibitory circuits. Pain inhibition and facilitation are both associated with increased neuronal activity in the same brainstem structures such as RVM or PAG, albeit in different cell types. With the currently available in vivo imaging modalities, activity in facilitatory ON and inhibitory OFF cells cannot be differentiated in humans due to their close proximity. Consequently, the demonstration of reduced brainstem activation in response to experimental thermal stimuli in subjects who experience migraines (73) is difficult to interpret because patients and controls rated the stimuli as equally intense, suggesting decreased brainstem activation did not change the intensity perception of the stimuli employed in the study. While the imaging finding would be in accordance with a dysfunction of pain inhibitory circuitry, more studies that provide a link to patients' symptoms, and thereby shed light on the functional significance of altered brainstem activation, are needed.

As discussed above, the anticipation of pain can activate similar structures as pain itself. However, the opposite has been observed for visceral pain: healthy subjects showed decreases of the BOLD fMRI signal in several regions (brainstem, IC, and ACC) during the anticipation of painful rectal distension (74). In contrast, such deactivations were absent in IBS patients. Although the interpretation of negative BOLD signals is not unambiguous, the data suggest that the absence of the negative BOLD signal during anticipation might be related to the patients' symptoms: correlation analyses suggested that the less deactivation a subject had, the higher the subject's negative affect scores and the lower their pain thresholds. Further, the degree of brainstem deactivation during anticipation was related to activation of the orbitofrontal cortex and rostral ACC during rectal distension, which might indicate that a pain inhibitory network was compromised in the patients. The notion of reduced pain inhibition due to a dysfunctional rostral ACC is potentially supported by a study in fibromyalgia patients (75): despite the same perceived pain intensity (which required lower stimulus intensities in the patients), control subjects showed more activation in the rostral ACC (and posterior thalamus) than patients.

Diffuse noxious inbibitory control. Counterirritation - or "pain inhibits pain" - is a phenomenon that has been known for centuries to decrease pain perception. Work in animals has identified the putative physiological basis, termed "diffuse noxious inhibitory control" (DNIC) (76). When an intense pain stimulus (the "conditioning" stimulus) is simultaneously applied to a remote body site, neural activity associated with the painful test stimulus is reduced both in wide-dynamic range as well as nociceptive-specific neurons of the spinal dorsal horn (reviewed in ref. 77). The fact that this phenomenon occurs in anesthetized animals implies that DNIC is separate from distraction or other cognitive operations requiring a conscious brain, although such processes might contribute to the perceptual analgesia induced by certain counterirritation protocols used in humans. Perhaps the biggest methodological problem in human studies occurs when the conditioning and the test stimuli are applied simultaneously, because this does not readily allow separation of DNIC from distraction. Nevertheless, a few well-controlled behavioral studies are available that indeed provide evidence that the DNIC system is impaired in chronic pain patients $(78,79)$. Human imaging studies have begun to describe brain correlates of the counterirritation phenomenon. Decreased activation evoked by the test stimulus has been demonstrated in healthy volunteers in several pain-related brain areas (including S1, ACC, thalamus, and posterior IC), in accordance with the observed perceptual analgesia (80). Sustained activation induced by the conditioning stimulus in the orbitofrontal cortex predicted the degree of analgesia, and consequently, this brain region probably plays a role in mediating counterirritation. However, sustained orbitofrontal cortex activation might reflect a combination of DNIC and distraction, which has been shown to engage the orbitofrontal cortex in close proximity (33), because the analysis described in the DNIC study was conducted for the period of simultaneous application of the two stimuli. Two studies thus far have used functional brain imaging to investigate counterirritation in pain patients $(53,81)$. In IBS patients, areas related to attention seemed less activated compared with control subjects, both during rectal stimulation (the test stimulus) as well as during the simultaneous application of the conditioning and the test stimulus (although no direct comparison was performed between patients and controls) (53). This might signify that patients paid less attention to the rectal stimulation (which can be expected to reduce pain and pain-related activation) irrespective of the presence of the conditioning stimulus, maybe because they attended to their clinical pain. Future imaging studies that exploit well-controlled DNIC designs will likely lead to more readily interpretable results.

\section{Structural brain alterations in chronic pain patients}

Individuals who suffer from long-term pain not only process pain differently, but also present with structural brain changes. Relatively recently, researchers have begun to apply diffusion-weighted 
A
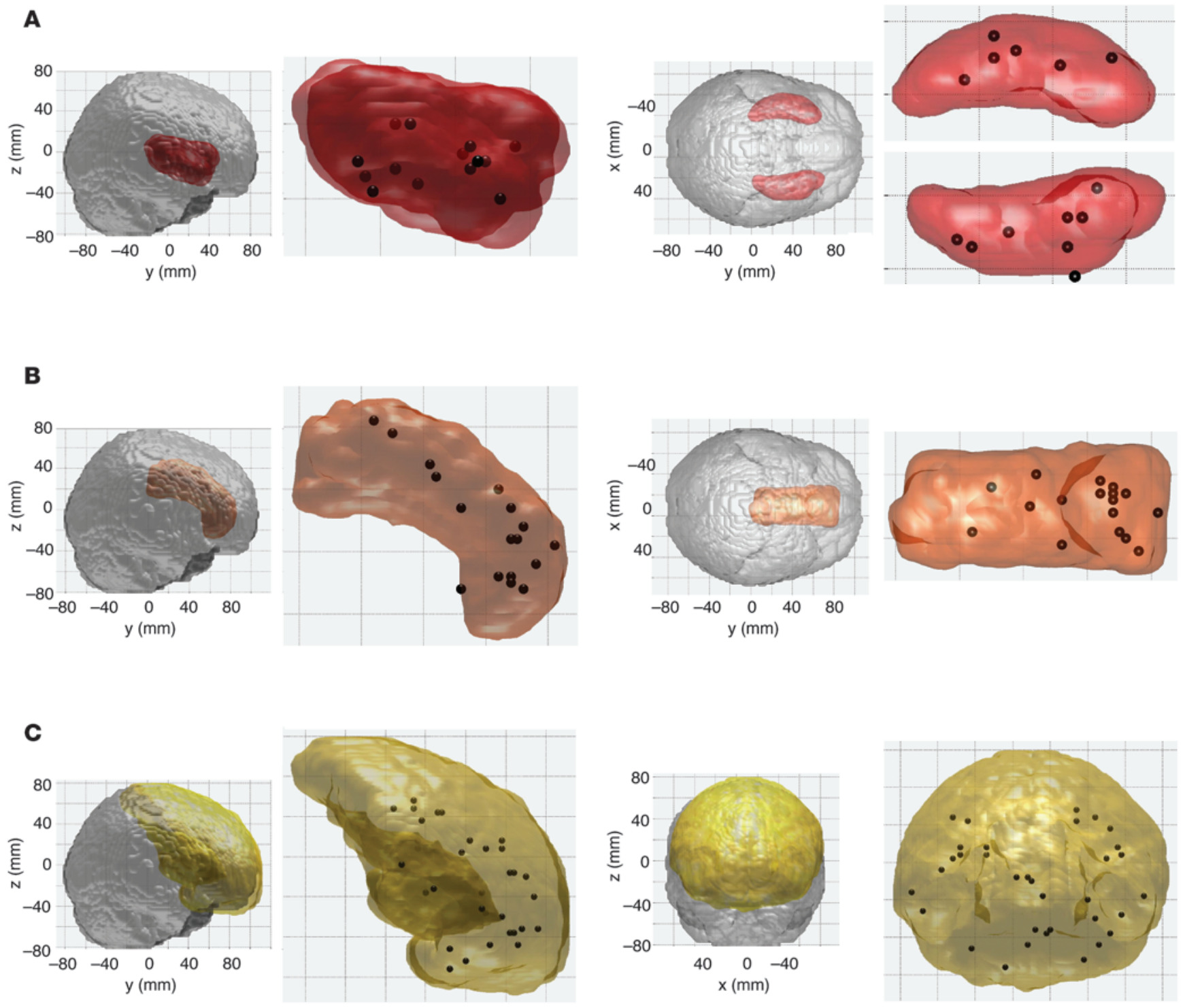

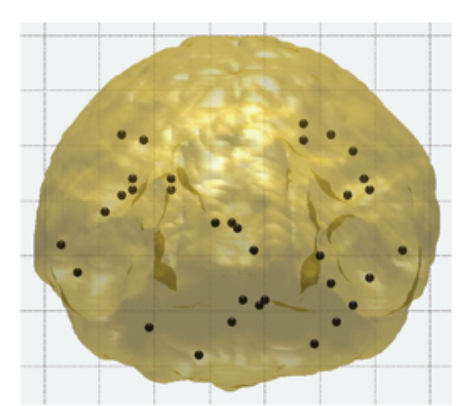

Figure 4

Localization of gray matter decreases in chronic pain conditions that have been reported for insula (A), ACC (B), and PFC (C) (for references, see text). Each sphere represents a peak of gray matter decrease. Note that more than one peak per region was included when reported in a study. Coordinates are in MNI standard stereotaxic space. $x$ refers to left-right (negative numbers in the left hemisphere, positive numbers in the right hemisphere). The anterior commissure corresponds to $0 \mathrm{~mm}$ in all three directions. In C, gray matter decreases are only depicted if they are located in the PFC, while the yellow volume encompasses all of frontal cortex, including precentral gyrus. Similarly, decreases in supplementary motor area and premotor cortex are not included. The volume for the insula (red) and frontal cortex (yellow) are based on the MNI Structural Atlas $(120,121)$, thresholded so that every voxel in the volume falls within the respective brain region (insula or frontal cortex) in at least $5 \%$ of individuals. The ACC volume (orange) is based on the Harvard-Oxford Cortical Structural Atlas, equally thresholded at $5 \%$. The MNI Structural Atlas and the Harvard-Oxford Cortical Structural Atlas were used as implemented in fslview of the fsl software suite (http://www.fmrib.ox.ac. $\mathrm{uk} / \mathrm{fs} \mathrm{l})$. The size of the spheres representing the localization of gray matter decreases is arbitrary.

imaging techniques, including DTI, to study white matter alterations in the brains of chronic pain patients. Geha and colleagues (17) found decreased diffusion directionality (fractional anisotropy) in the cingulum of patients with complex regional pain syndrome (CRPS), which might possibly indicate decreased tract myelination or reduced parallel fiber organization. Fewer white matter connections were found to originate in the patients from this spot of altered diffusion compared with control subjects. Further, the ventral medial PFC, an area of decreased gray matter, showed an altered anatomical connectivity pattern, adding further evidence to impaired white matter connectivity in the CRPS patients of this study (17). Some clinical significance of decreased diffusion directionality was provided by a study in fibromyalgia patients that demonstrated a relationship between decreased fractional anisotropy in the thalamocortical tract and the degree of stiffness (82). Schmitz and colleagues assessed the concentration of white matter in migraine patients, rather than investigating diffusion properties of white matter (83). They found that patients 
with a high attack frequency had decreased white matter concentration of frontal and parietal areas (83), perhaps indicating that migraine attacks lead to white matter damage.

In contrast to the scarcity of studies investigating white matter, gray matter alterations in chronic pain patients have been frequently studied in recent years, and this literature supports the hypothesis that pain or prolonged nociceptive input leads to structural alterations. Although it might seem likely that different pain conditions are associated with unique sets of structural alterations, such comparative data are not yet available. Seemingly similar gray matter changes have been described across different chronic pain conditions, including fibromyalgia, migraine, or osteoarthritis. Consequently, we do not differentiate between etiologies in the following discussion. Regarding the direction of structural alterations, decreases in gray matter dominate the picture, although some studies have reported an increased concentration of gray matter, either exclusively (84) or alongside gray matter decreases (85-88). Decreases have mainly been described for important pain processing or modulatory regions such as the ACC, IC, thalamus, and frontal cortex $(17,83,85,86,88-103)$ as well as the (para-)hippocampus, which is thought to be particularly vulnerable to the effects of stress $(82,93,97,98,103)$ (Figure 4). Several studies observed greater gray matter decreases with longer pain duration $(17,83,89,92-94,97-99)$, which might indicate that gray matter decreases are a consequence of living with pain, at least in these instances. The concept of pain or prolonged nociceptive input leading to decreased gray matter is supported by two lines of evidence. First, two longitudinal studies in patients (one study following successful hip replacement for osteoarthritis [ref. 95], the other after spontaneous resolution of post-traumatic headache [ref. 85]) suggest that gray matter concentrations return to baseline levels when the pain disappears. Second, a longitudinal MRI study in a rat model of neuropathic pain (spared nerve injury) demonstrated the occurrence of prefrontal gray matter alterations several months after pain induction by surgery (104). This study provides another very interesting piece of information: the gray matter alterations in the PFC coincided with the development of anxiety-like behavior (104), emphasizing behavioral consequences of prolonged pain states. Although the mechanisms underlying gray matter alterations in chronic pain are currently unknown, a recently conducted study suggests a possibility. DaSilva and colleagues observed that reduced thickness of sensorimotor cortex in patients with trigeminal neuralgia was co-localized with activation related to provocation of their dynamic mechanical allodynia (105). This suggests that excessive nociceptive input might lead to gray matter reductions. This would be similar to the neurotoxicity hypothesis of depression (reviewed in ref. 106), which postulates that an interaction between elevated levels of circulating glucocorticoids and excitatory neural activity is a major factor in long-term cerebral atrophy. However, it should be emphasized that conventional MRI cannot determine the histopathology underlying gray matter changes, including the affected cell type. Therefore, gray matter alterations demonstrated in chronic pain could be caused by changes in neuronal elements such as dendrites or synapses, glial cells, or even blood vessels or water content. To advance our understanding of the nature of gray matter alterations, future studies will have to complement anatomical MRI with other imaging techniques. One magnetic resonance-based technique that can be used to yield complementary information is proton magnetic resonance spectroscopy $\left({ }^{1} \mathrm{H}-\mathrm{MRS}\right) .{ }^{1} \mathrm{H}-\mathrm{MRS}$ exploits the fact that the resonance frequency of a proton is influenced by its exact biochemical environment, meaning that the signals from individual metabolites can be differentiated. Metabolites that can be measured using this technique include the neuronal marker $\mathrm{N}$-acetylaspartate (NAA) $(107,108)$, choline-containing compounds, which indicate membrane turnover and cellular density, and glutamate/ glutamine. Studies using this technique in the context of pain are described below.

\section{Cerebral biochemical alterations associated with chronic pain}

A preliminary study of 20 healthy elderly subjects with varying levels of chronic pain found pain severity to be related to lower NAA levels in the hippocampus as well as to reduced hippocampal volumes (109). This suggests that smaller hippocampi might be partly explained by a decrease in neuronal tissue. As indicated by anatomical studies, the hippocampus appears to be a brain region that is frequently affected by pain. Two studies have observed decreased hippocampal NAA levels in fibromyalgia $(110,111)$, and one of those also reported an inverse relationship between NAA and fibromyalgia severity (as measured by the fibromyalgia impact questionnaire [FIQ]) (111). One patient from this study underwent successful pharmacological treatment, and after 28 weeks the FIQ score was down to 8 (from 52) and NAA in the right hippocampus seemed to have returned to normal levels (112). As discussed above, reduced gray matter in chronic pain has been frequently observed in the thalamus and frontal cortex. To the best of our knowledge, no study has yet reported on NAA in these areas while measuring gray matter in the same patients, but it should be noted that NAA reductions have been described in the thalamus (113, 114) and dorsolateral PFC (115-117) of chronic pain patients.

In light of a potential analogy to neurotoxicity observed in other disease entities and related to excessive levels of excitatory neurotransmitters, it is interesting to note that higher glutamate levels in the IC have been reported during the interictal phase in migraine patients (118). In patients with fibromyalgia, glutamate levels in the insula decreased after treatment, and a striking relationship was observed between decreased glutamate and improved clinical pain as well as increased pressure pain thresholds (119).

\section{Conclusions}

Modern imaging techniques have permitted rapid progress in our understanding of networks in the brain related to pain processing and those related to different types of pain modulation, and the dramatic advances in both imaging equipment and analytical techniques will allow us to delve even more deeply into the impact of chronic pain on the brain. The first modern fMRI experiments in humans were conducted on 1.5-tesla scanners; now, 3-tesla is the norm, and 7-tesla machines are being adopted for anatomical and functional imaging. With these new machines come increased spatial and temporal resolution and a greater ability to detect small signals in the brain.

The neural basis of placebo analgesia is being deciphered, and we have begun to directly evaluate functional and anatomical connectivity of these circuits. Work is being done to improve our ability to successfully image pain-related activity in the spinal cord of humans. Perhaps the most exciting and revealing data have come from studies of chronic pain patients, in which imaging has provided strong evidence that pain leads to brain changes that could have functional significance. These technologies have indi- 
cated that chronic pain patients have altered pain modulatory circuits, both at forebrain and brainstem levels. Further, significant structural abnormalities have been revealed in a number of brain regions, particularly those involved in pain modulation. Finally, imaging studies are beginning to identify biochemical alterations associated with chronic pain that could underlie neurotoxicity caused by long-term activation. The future is bright for what brain imaging can contribute to our understanding of pain. Especially in combination with cellular, genetic, and molecular approaches, imaging techniques might have a major impact in the diagnosis and differentiation of chronic pain problems and the evaluation of the effectiveness of therapeutic interventions.

\section{Acknowledgments}

P. Schweinhardt is supported by the Canadian Institutes for Health Research (CIHR), Fonds de la recherche en santé (FRSQ), the International Association for the Study of Pain (IASP), and the Louise and Alan Edwards Foundation. M.C. Bushnell is supported by the CIHR, FRSQ, American Fibromyalgia Syndrome Association (AFSA), and Pfizer Canada.

Address correspondence to: M. Catherine Bushnell, Alan Edwards Centre for Research on Pain, McGill University, 3640 University Street, Room M19, Montreal, Quebec, Canada H3A 2B2. Phone: 514.398. 3493; Fax: 514.398.7464; E-mail: catherine.bushnell@mcgill.ca.
1. Penfield W, Rasmussen T. The Cerebral Cortex of Man. New York, New York, USA: The Macmillan Company; 1955.

2. Apkarian AV, Stea RA, Manglos SH, Szeverenyi NM, King RB, Thomas FD. Persistent pain inhibits contralateral somatosensory cortical activity in humans. Neurosci Lett. 1992;140(2):141-147.

3. Jones AK, Brown WD, Friston KJ, Qi LY, Frackowiak RS. Cortical and subcortical localization of response to pain in man using positron emission tomography. Proc Biol Sci. 1991;244(1309):39-44.

4. Talbot JD, Marrett S, Evans AC, Meyer E, Bush nell MC, Duncan GH. Multiple representations of pain in human cerebral cortex. Science. 1991; 251(4999):1355-1358.

5. Tracey I, Johns E. The pain matrix: reloaded or reborn as we image tonic pain using arterial spin labelling. Pain. 2010;148(3):359-360.

6. Bushnell MC, Apkarian AV. Representation of pain in the brain. In: McMahon SB, Koltzenburg M, eds Textbook of Pain. 5th ed. Philadelphia, Pennsylvania, USA: Elsevier Churchill Livingstone; 2005:107-124.

7. Apkarian AV, Bushnell MC, Treede RD, Zubieta JK. Human brain mechanisms of pain perception and regulation in health and disease. Eur J Pain. 2005;9(4):463-484.

8. Price DD. Psychological and neural mechanisms of the affective dimension of pain. Science. 2000; 288(5472):1769-1772.

9. Baliki MN, et al. Chronic pain and the emotional brain: specific brain activity associated with spontaneous fluctuations of intensity of chronic back pain. J Neurosci. 2006;26(47):12165-12173.

10. Tracey I, Johns E. The pain matrix: reloaded or reborn as we image tonic pain using arterial spin labelling. Pain. 2010;148(3):359-360.

11. Wang J, Aguirre GK, Kimberg DY, Roc AC, Li L, Detre JA. Arterial spin labeling perfusion fMRI with very low task frequency. Magn Reson Med. 2003;49(5):796-802.

12. Owen DG, Clarke CF, Ganapathy S, Prato FS, St Lawrence KS. Using perfusion MRI to measure the dynamic changes in neural activation associated with tonic muscular pain. Pain. 2010;148(3):375-386.

13. Villemure C, Bushnell MC. Mood influences supraspinal pain processing separately from attention. J Neurosci. 2009;29(3):705-715.

14. Ploner M, Lee MC, Wiech K, Bingel U, Tracey I. Prestimulus functional connectivity determines pain perception in humans. Proc Natl Acad Sci US A. 2010;107(1):355-360.

15. Conturo TE, et al. Tracking neuronal fiber pathways in the living human brain. Proc Natl Acad Sci US A. 1999;96(18):10422-10427.

16. Hadjipavlou G, Dunckley P, Behrens TE, Tracey I. Determining anatomical connectivities between cortical and brainstem pain processing regions in humans: A diffusion tensor imaging study in healthy controls. Pain. 2006;123(1-2):169-178.

17. Geha PY, Baliki MN, Harden RN, Bauer WR, Parrish TB, Apkarian AV. The brain in chronic CRPS pain: abnormal gray-white matter interactions in emotional and autonomic regions. Neuron. 2008; 60(4):570-581.

18. Giove F, Garreffa G, Giulietti G, Mangia S, Colonnese C, Maraviglia B. Issues about the fMRI of the human spinal cord. Magn Reson Imaging. 2004:22(10):1505-1516.

19. Brooks JC, et al. Physiological noise modelling for spinal functional magnetic resonance imaging studies. Neuroimage. 2008;39(2):680-692.

20. Eippert F, Finsterbusch J, Bingel U, Buchel C. Direct evidence for spinal cord involvement in placebo analgesia. Science. 2009;326(5951):404.

21. Ghazni NF, Cahill CM, Stroman PW. Tactile sensory and pain networks in the human spinal cord and brain stem mapped by means of functional MR imaging. AJNR Am J Neuroradiol. 2010;31(4):661-667.

22. Malisza KL, Stroman PW. Functional imaging of the rat cervical spinal cord. J Magn Reson Imaging. 2002;16(5):553-558.

23. Zhao F, et al. Pain $\mathrm{AMRI}$ in rat cervical spinal cord: an echo planar imaging evaluation of sensitivity of BOLD and blood volume-weighted PMRI. Neuroimage. 2009;44(2):349-362.

24. Benedetti F, Mayberg HS, Wager TD, Stohler CS, Zubieta JK. Neurobiological mechanisms of the placebo effect. J Neurosci. 2005;25(45):10390-10402.

25. Villemure C, Bushnell MC. Cognitive modulation of pain: how do attention and emotion influence pain processing? Pain. 2002;95(3):195-199.

26. Villemure C, Schweinhardt P. Supraspinal pain processing: distinct roles of emotion and attention. Neuroscientist. 2010;16(3):276-284.

27. Bantick SJ, Wise RG, Ploghaus A, Clare S, Smith $\mathrm{SM}$, Tracey I. Imaging how attention modulates pain in humans using functional MRI. Brain. 2002; 125(pt 2):310-319

28. Brooks JC, Nurmikko TJ, Bimson WE, Singh KD, Roberts N. fMRI of thermal pain: effects of stimulus laterality and attention. Neuroimage. 2002; 15(2):293-301.

29. Longe SE, et al. Counter-stimulatory effects on pain perception and processing are significantly altered by attention: an fMRI study. Neuroreport. 2001; 12(9):2021-2025.

30. Valet M, et al. Distraction modulates connectivity of the cingulo-frontal cortex and the midbrain during pain--an fMRI analysis. Pain. 2004;109(3):399-408.

31. Wiech $\mathrm{K}$, et al. Modulation of pain processing in hyperalgesia by cognitive demand. Neuroimage. 2005;27(1):59-69.

32. Frankenstein UN, Richter W, McIntyre MC, Remy F. Distraction modulates anterior cingulate gyrus activations during the cold pressor test. Neuroimage. 2001;14(4):827-836.

33. Petrovic P, Petersson KM, Ghatan PH, StoneElander S, Ingvar M. Pain-related cerebral activation is altered by a distracting cognitive task. Pain. 2000;85(1-2):19-30.

34. Tracey I, et al. Imaging attentional modulation of pain in the periaqueductal gray in humans. $\mathrm{JNeu}$ - rosci. 2002;22(7):2748-2752.

35. De Wied M, Verbaten MN. Affective pictures processing, attention, and pain tolerance. Pain. 2001; 90(1-2):163-172.

36. Marchand S, Arsenault P. Odors modulate pain perception: a gender-specific effect. Physiol Behav. 2002;76(2):251-256.

37. Meagher MW, Arnau RC, Rhudy JL. Pain and emotion: effects of affective picture modulation. Psychosom Med. 2001;63(1):79-90.

38. Wiech K, Tracey I. The influence of negative emotions on pain: behavioral effects and neural mechanisms. Neuroimage. 2009;47(3):987-994.

39. Zelman DC, Howland EW, Nichols SN, Cleeland CS. The effects of induced mood on laboratory pain. Pain. 1991;46(1):105-111.

40. Phillips ML, et al. The effect of negative emotional context on neural and behavioural responses to oesophageal stimulation. Brain. 2003; 126(pt 3):669-684.

41. Berna C, Leknes S, Holmes EA, Edwards RR, Goodwin GM, Tracey I. Induction of depressed mood disrupts emotion regulation neurocircuitry and enhances pain unpleasantness. Biol Psychiatry. 2010; 67(11):1083-1090.

42. Fields H. State-dependent opioid control of pain. Nat Rev Neurosci. 2004;5(7):565-575.

43. Corbetta M, Shulman GL. Control of goal-directed and stimulus-driven attention in the brain. Nat Rev Neurosci. 2002;3(3):201-215.

44. Beydoun A, Morrow TJ, Shen JF, Casey KL. Variability of laser-evoked potentials: attention, arousal and lateralized differences. Electroencephalogr Clin Neurophysiol. 1993;88(3):173-181.

45. Fairhurst M, Wiech K, Dunckley P, Tracey I. Anticipatory brainstem activity predicts neural processing of pain in humans. Pain. 2007;128(1-2):101-110.

46. Hsieh JC, Stone-Elander S, Ingvar M. Anticipatory coping of pain expressed in the human anterior cingulate cortex: a positron emission tomography study. Neurosci Lett. 1999;262(1):61-64.

47. Jensen J, McIntosh AR, Crawley AP, Mikulis DJ, Remington G, Kapur S. Direct activation of the ventral striatum in anticipation of aversive stimuli. Neuron. 2003;40(6):1251-1257.

48. Lopez-Sola M, et al. Dynamic assessment of the right lateral frontal cortex response to painful stimulation. Neuroimage. 2010;50(3):1177-1187.

49. Ploghaus A, et al. Dissociating pain from its anticipation in the human brain. Science. 1999; 284(5422):1979-1981.

50. Porro CA, et al. Does anticipation of pain affect cortical nociceptive systems? J Neurosci. 2002; 22(8):3206-3214

51. Sawamoto N, et al. Expectation of pain enhances responses to nonpainful somatosensory stimulation in the anterior cingulate cortex and parietal operculum/posterior insula: an event-related functional magnetic resonance imaging study. J Neurosci. 2000; 20(19):7438-7445.

52. Burgmer M, et al. Fibromyalgia unique tempo- 
ral brain activation during experimental pain: a controlled fMRI Study. J Neural Transm. 2010; 117(1):123-131.

53. Song GH, Venkatraman V, Ho KY, Chee MW, Yeoh KG, Wilder-Smith CH. Cortical effects of anticipation and endogenous modulation of visceral pain assessed by functional brain MRI in irritable bowel syndrome patients and healthy controls. Pain. 2006; 126(1-3):79-90.

54. Strigo IA, Simmons AN, Matthews SC, Craig AD, Paulus MP. Association of major depressive disorder with altered functional brain response during anticipation and processing of heat pain. Arch Gen Psychiatry. 2008;65(11):1275-1284.

55. Wager TD, et al. Placebo-induced changes in FMRI in the anticipation and experience of pain. Science. 2004;303(5661):1162-1167.

56. Watson A, et al. Placebo conditioning and placebo analgesia modulate a common brain network during pain anticipation and perception. Pain. 2009; 145(1-2):24-30.

57. Wager TD, Scott DJ, Zubieta JK. Placebo effects on human mu-opioid activity during pain. Proc Natl Acad Sci U S A. 2007;104(26):11056-11061.

58. Eippert F, et al. Activation of the opioidergic descending pain control system underlies placebo analgesia. Neuron. 2009;63(4):533-543.

59. Derbyshire SW, et al. Cerebral responses to noxious thermal stimulation in chronic low back pain patients and normal controls. Neuroimage. 2002; 16(1):158-168

60. Gracely RH, Petzke F, Wolf JM, Clauw DJ. Functional magnetic resonance imaging evidence of augmented pain processing in fibromyalgia. Arthritis Rheum. 2002;46(5):1333-1343.

61. Lawal A, Kern M, Sidhu H, Hofmann C, Shaker R. Novel evidence for hypersensitivity of visceral sensory neural circuitry in irritable bowel syndrome patients. Gastroenterology. 2006;130(1):26-33.

62. Naliboff BD, et al. Cerebral activation in patients with irritable bowel syndrome and control subjects during rectosigmoid stimulation. Psychosom Med. 2001;63(3):365-375.

63. Pukall CF, Strigo IA, Binik YM, Amsel R, Khalife S, Bushnell MC. Neural correlates of painful genital touch in women with vulvar vestibulitis syndrome. Pain. 2005;115(1-2):118-127.

64. Schweinhardt P, et al. An fMRI study of cerebral processing of brush-evoked allodynia in neuropathic pain patients. Neuroimage. 2006;32(1):256-265.

65. Fields HL, Heinricher MM. Anatomy and physiology of a nociceptive modulatory system. Philos Trans $R$ Soc Lond B Biol Sci. 1985;308(1136):361-374.

66. Porreca F, Ossipov MH, Gebhart GF. Chronic pain and medullary descending facilitation. Trends Neurosci. 2002;25(6):319-325.

67. Lee MC, Zambreanu L, Menon DK, Tracey I. Identifying brain activity specifically related to the maintenance and perceptual consequence of central sensitization in humans. J Neurosci. 2008; 28(45):11642-11649.

68. Gwilym SE, et al. Psychophysical and functional imaging evidence supporting the presence of central sensitization in a cohort of osteoarthritis patients. Arthritis Rheum. 2009;61(9):1226-1234.

69. Freynhagen R, Baron R, Gockel U, Tölle TR. painDETECT: a new screening questionnaire to identify neuropathic components in patients with back pain. Curr Med Res Opin. 2006;22(10):1911-1920.

70. Schweinhardt P, Kalk N, Wartolowska K, Chessell I Wordsworth P, Tracey I. Investigation into the neural correlates of emotional augmentation of clinical pain. Neuroimage. 2008;40(2):759-766.

71. Ploghaus A, et al. Exacerbation of pain by anxiety is associated with activity in a hippocampal network. J Neurosci. 2001;21(24):9896-9903.

72. Mayer EA, et al. Differences in brain responses to visceral pain between patients with irritable bowel syndrome and ulcerative colitis. Pain. 2005; 115(3):398-409.

73. Moulton EA, Burstein R, Tully S, Hargreaves R, Becerra L, Borsook D. Interictal dysfunction of a brainstem descending modulatory center in migraine patients. PLoS One. 2008;3(11):e3799.

74. Berman SM, et al. Reduced brainstem inhibition during anticipated pelvic visceral pain correlates with enhanced brain response to the visceral stimulus in women with irritable bowel syndrome. J Neurosci. 2008;28(2):349-359.

75. Jensen $\mathrm{KB}$, et al. Evidence of dysfunctional pain inhibition in Fibromyalgia reflected in rACC during provoked pain. Pain. 2009;144(1-2):95-100

76. Villanueva L, Le Bars D. The activation of bulbospinal controls by peripheral nociceptive inputs: diffuse noxious inhibitory controls. Biol Res. 1995; 28(1):113-125

77. Le Bars D. The whole body receptive field of dorsal horn multireceptive neurones. Brain Res Brain Res Rev. 2002;40(1-3):29-44.

78. de Souza JB, Potvin S, Goffaux P, Charest J, Marchand $\mathrm{S}$. The deficit of pain inhibition in fibromyalgia is more pronounced in patients with comorbid depressive symptoms. Clin J Pain. 2009;25(2):123-127.

79. Julien N, Goffaux P, Arsenault P, Marchand S. Widespread pain in fibromyalgia is related to a deficit of endogenous pain inhibition. Pain. 2005; 114(1-2):295-302.

80. Piche M, Arsenault M, Rainville P. Cerebral and cerebrospinal processes underlying counterirritation analgesia. J Neurosci. 2009;29(45):14236-14246.

81. Wilder-Smith CH, Schindler D, Lovblad K, Redmond SM, Nirkko A. Brain functional magnetic resonance imaging of rectal pain and activation of endogenous inhibitory mechanisms in irritable bowel syndrome patient subgroups and healthy controls. Gut. 2004;53(11):1595-1601.

82. Lutz J, et al. White and gray matter abnormalities in the brain of patients with fibromyalgia: a diffusion-tensor and volumetric imaging study. Arthritis Rheum. 2008;58(12):3960-3969.

83. Schmitz N, et al. Attack frequency and disease duration as indicators for brain damage in migraine. Headache. 2008;48(7):1044-1055.

84. Schweinhardt P, Kuchinad A, Pukall CF, Bushnell MC. Increased gray matter density in young women with chronic vulvar pain. Pain. 2008;140(3):411-419.

85 . Obermann $M$, et al. Gray matter changes related to chronic posttraumatic headache. Neurology. 2009 73(12):978-983.

86. Schmidt-Wilcke T, et al. Affective components and intensity of pain correlate with structural differences in gray matter in chronic back pain patients. Pain. 2006;125(1-2):89-97.

87. Schmidt-Wilcke T, et al. Striatal grey matter increase in patients suffering from fibromyalgia -a voxel-based morphometry study. Pain. 2007 132(suppl 1):S109-S116.

88. Seminowicz DA, et al. Regional gray matter density changes in brains of patients with irritable bowel syndrome. Gastroenterology. 2010;139(1):48-57.e2.

89. Apkarian AV, et al. Chronic back pain is associated with decreased prefrontal and thalamic gray matter density. J Neurosci. 2004;24(46):10410-10415

90. Burgmer M, et al. Decreased gray matter volumes in the cingulo-frontal cortex and the amygdala in patients with fibromyalgia. Psychosom Med. 2009; 71(5):566-573

91. Davis KD, Pope G, Chen J, Kwan CL, Crawley AP, Diamant NE. Cortical thinning in IBS: implications for homeostatic, attention, and pain processing. Neurology. 2008;70(2):153-154.

92. Kim JH, et al. Regional grey matter changes in patients with migraine: a voxel-based morphometry study. Cephalalgia. 2008;28(6):598-604.

93. Kuchinad A, Schweinhardt P, Seminowicz DA, Wood PB, Chizh BA, Bushnell MC. Accelerated brain gray matter loss in fibromyalgia patients: premature aging of the brain? J Neurosci. 2007;27(15):4004-4007.

94. Rocca MA, et al. Brain gray matter changes in migraine patients with T2-visible lesions: a 3-T MRI study. Stroke. 2006;37(7):1765-1770.

95. Rodriguez-Raecke R, Niemeier A, Ihle K, Ruether W, May A. Brain gray matter decrease in chronic pain is the consequence and not the cause of pain. J Neurosci. 2009;29(44):13746-13750.

96. Schmidt-Wilcke T, Ganssbauer S, Neuner T, Bogdahn U, May A. Subtle grey matter changes between migraine patients and healthy controls. Cephalalgia. 2008;28(1):1-4.

97. Schmidt-Wilcke T, et al. Gray matter decrease in patients with chronic tension type headache. $\mathrm{Neu}$ rology. 2005;65(9):1483-1486

98. Valet M, et al. Patients with pain disorder show gray-matter loss in pain-processing structures: a voxel-based morphometric study. Psychosom Med. 2009;71(1):49-56

99. Valfre W, Rainero I, Bergui M, Pinessi L. Voxel-based morphometry reveals gray matter abnormalities in migraine. Headache. 2008;48(1):109-117.

100.Vartiainen $\mathrm{N}$, et al. Changes in brain function and morphology in patients with recurring herpes simplex virus infections and chronic pain. Pain. 2009;144(1-2):200-208.

101.Schmidt-Wilcke T, Hierlmeier S, Leinisch E. Altered regional brain morphology in patients with chronic facial pain [published online ahead of print March 5, 2010]. Headache doi: 10.1111/j.15264610.2010 .01637$.

102.Schmitz N, et al. Frontal lobe structure and executive function in migraine patients. Neurosci Lett. 2008; 440(2):92-96

103. Wood PB, Glabus MF, Simpson R, Patterson JC 2nd. Changes in gray matter density in fibromyalgia: correlation with dopamine metabolism. J Pain. 2009;10(6):609-618.

104.Seminowicz DA, Laferriere AL, Millecamps M, Yu JS, Coderre TJ, Bushnell MC. MRI structural brain changes associated with sensory and emotional function in a rat model of long-term neuropathic pain. Neuroimage. 2009;47(3):1007-1014.

105.DaSilva AF, Becerra L, Pendse G, Chizh B, Tully S, Borsook D. Colocalized structural and functional changes in the cortex of patients with trigeminal neuropathic pain. PLoS One. 2008;3(10):e3396.

106. McEwen BS. Effects of adverse experiences for brain structure and function. Biol Psychiatry. 2000; 48(8):721-731.

107. Moffett JR, Williamson LC, Neale JH, Palkovits M, Namboodiri MA. Effect of optic nerve transection on $\mathrm{N}$-acetylaspartylglutamate immunoreactivity in the primary and accessory optic projection systems in the rat. Brain Res. 1991;538(1):86-94.

108. Simmons ML, Frondoza CG, Coyle JT. Immunocytochemical localization of $\mathrm{N}$-acetyl-aspartate with monoclonal antibodies. Neuroscience. 1991; 45(1):37-45.

109.Zimmerman ME, Pan JW, Hetherington HP, Lipton ML, Baigi K, Lipton RB. Hippocampal correlates of pain in healthy elderly adults: a pilot study. Neurology. 2009;73(19):1567-1570.

110.Emad Y, Ragab Y, Zeinhom F, El-Khouly G, AbouZeid A, Rasker JJ. Hippocampus dysfunction may explain symptoms of fibromyalgia syndrome. A study with single-voxel magnetic resonance spectroscopy. J Rheumatol. 2008;35(7):1371-1377.

111. Wood PB, Ledbetter CR, Glabus MF, Broadwell LK, Patterson JC 2nd. Hippocampal metabolite abnormalities in fibromyalgia: correlation with clinical features. J Pain. 2009;10(1):47-52.

112. Wood PB, Ledbetter CR, Patterson JC 2nd. Changes in hippocampal metabolites after effective treatment for fibromyalgia: a case study. Clin J Pain. 2009; 25(9):810-814

113.Fukui S, Matsuno M, Inubushi T, Nosaka S. 
$\mathrm{N}$-Acetylaspartate concentrations in the thalami of neuropathic pain patients and healthy comparison subjects measured with (1)H-MRS. Magn Reson Imaging. 2006;24(1):75-79.

114.Sorensen L, Siddall PJ, Trenell MI, Yue DK. Differences in metabolites in pain-processing brain regions in patients with diabetes and painful neuropathy. Diabetes Care. 2008;31(5):980-981.

115. Grachev ID, Fredrickson BE, Apkarian AV. Abnormal brain chemistry in chronic back pain: an in vivo proton magnetic resonance spectroscopy study. Pain. 2000;89(1):7-18.
116.Grachev ID, Fredrickson BE, Apkarian AV. Brain chemistry reflects dual states of pain and anxiety in chronic low back pain. J Neural Transm. 2002; 109(10):1309-1334.

117. Grachev ID, Thomas PS, Ramachandran TS. Decreased levels of $\mathrm{N}$-acetylaspartate in dorsolateral prefrontal cortex in a case of intractable severe sympathetically mediated chronic pain (complex regional pain syndrome, type I). Brain Cogn. 2002; 49(1):102-113.

118. Prescot A, et al. Excitatory neurotransmitters in brain regions in interictal migraine patients. $\mathrm{Mol}$
Pain. 2009;5:34

119. Harris RE, et al. Dynamic levels of glutamate within the insula are associated with improvements in multiple pain domains in fibromyalgia. Arthritis Rheum. 2008;58(3):903-907.

120.Collins DL, Holmes CJ, Peters TM, Evans A. Automatic 3-D model-based neuroanatomical segmentation. Hum Brain Mapp. 1995;3(3):190-208.

121. Mazziotta J, et al. A probabilistic atlas and reference system for the human brain: International Consortium for Brain Mapping (ICBM). Philos Trans R Soc Lond B Biol Sci. 2001;356(1412):1293-1322. 